\title{
Finite amplitude instability in Takens-Bogdanov-type dynamical systems
}

\author{
X. Z. Tang* \\ Princeton Plasma Physics Laboratory, Princeton University, Princeton, NJ 08543
}

(December 2, 1998)

\begin{abstract}
A weakly nonlinear theory is given to treat the onset of a finite amplitude instability in a Takens-Bogdanov-type dynamical system. The theory is based on an asymptotic analysis.

PACS numbers: 64.60.Cn, 47.20.Ky, 47.20.Ft, 47.27.Cn
\end{abstract}

*Email: xtang@pppl.gov 


\section{INTRODUCTION}

The purpose of this paper is to present a weakly nonlinear theory that describes the onset of a finite amplitude instability in Takens-Bogdanov-type dynamical systems. The principle mathematical tool is an asymptotic analysis. By weakly, we mean a theory that is only able to treat the onset of the instability. The eventual saturation of the finite amplitude instability is usually case-dependent and requires more powerful methods.

\section{A. Finite amplitude instability}

An intuitive understanding of a finite amplitude instability can be obtained by making an analogy with the motion of a ball in a one dimensional well, figure 1 . The bottom of the well is made convex so it is a stable equilibrium state for the ball, both linearly and nonlinearly (Lyapunov stable). If there is friction between the contact surfaces of the ball and the well, the bottom of the well is asymptotically stable. A nonlinear system can depart from an asymptotically stable equilibrium indefinitely if there is a finite amplitude perturbation with sufficiently large amplitude $\sigma_{c}$. In the case of a ball in a well, figure 1 , the critical perturbation is given by the shape of the well, or in general terms, by the nonlinearity (greater than quadratic) in the 'potential' function. This is called a finite amplitude instability and it is characterized by the magnitude of $\sigma_{c}$.

A simple mathematical example is a first-order ordinary differential equation,

$$
\frac{d x}{d t}=-\epsilon x+x^{N}, \quad 0 \leq \epsilon<1
$$

with $N$ an integer greater than or equal to two. Obviously $x=0$ is a linearly stable equilibrium state. If $\epsilon>0, x=0$ is asymptotically stable. But the system does have a finite amplitude instability

$$
\sigma_{c}=\epsilon^{\alpha}, \text { with } \alpha=\frac{1}{N-1} \leq 1
$$

In dynamical systems with dimension higher than one, the critical perturbation usually has a directional dependence. In the theory of dissipative dynamical systems, the 


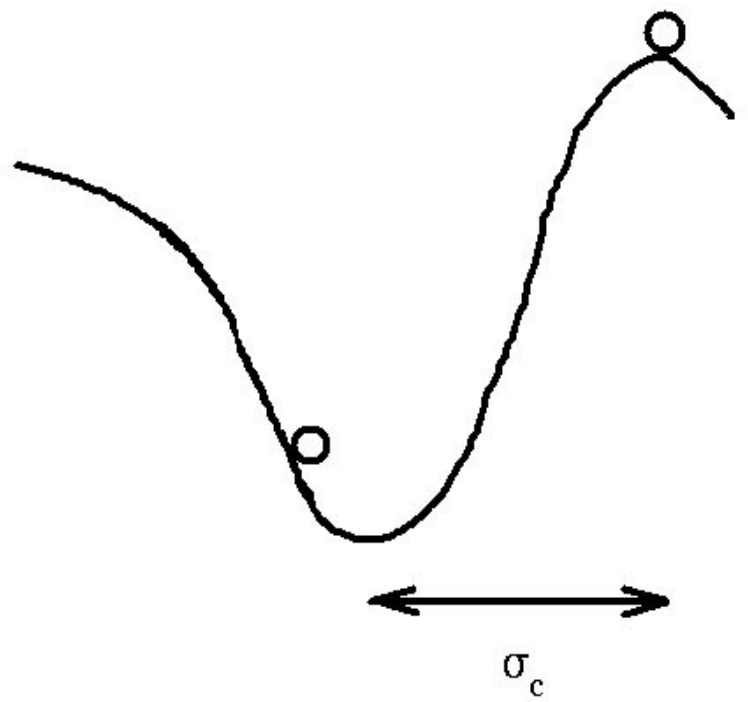

FIG. 1. Motion of a ball in a one dimensional frictional well. The bottom of the well is an asymptotically stable state for the ball. The system has a finite amplitude instability with critical perturbation $\sigma_{c}$.

hyper-surface defined by $\sigma_{c}$ is called the basin boundary of attraction for the linearly stable equilibrium. In the language of stability analysis, it is called a (nonlinear) finite amplitude instability. For the purpose of stability analysis, one usually simplifies the problem by concentrating on the smallest critical perturbation since that is the lowest bound to drive the system away from the equilibrium. The scaling relationship between the smallest critical perturbation $\sigma_{c}$ and the linear stability parameter $\epsilon$,

$$
\sigma_{c} \propto \epsilon^{\alpha}
$$

is of primary interest to assess the importance of a finite amplitude instability in a physical application. If not directly referred to as the basin boundary of attraction, $\sigma_{c}$ denotes the smallest critical perturbation amplitude in the multi-dimensional case.

Definition I.1 If the scaling exponent $\alpha \leq 1$, one has a normal finite amplitude instability. If $\alpha>1$, one has an anomalous finite amplitude instability. 
The greater the scaling exponent $\alpha$, the greater practical importance for the finite amplitude instability to induce a transition of states in a noisy environment. The possible existence of an anomalous scaling relationship is what makes the study of finite amplitude instability practically relevant and interesting.

\section{B. Takens-Bogdanov-type dynamical systems}

The definition of a Takens-Bogdanov-type dynamical system can be made precise by following the usual stability analysis of a dynamical system around an equilibrium point. Consider the stability of an $N$-dimensional autonomous dynamical system in a vector space with orthonormal basis $\hat{z}_{i}$ s,

$$
d z / d t=F(z), z \in \Re^{N} \text { and } F \in \Re^{N},
$$

around an equilibrium point $z_{e}, F\left(z_{e}\right)=0$. Define $y=z-z_{e}, y \in \Re^{N}$, and Taylor expand $F(z)$ in the neighborhood of the equilibrium $z_{e}$, the $n$th component of equation (1) becomes

$$
d y_{n} / d t=B_{n i} y_{i}+C_{n i j}^{(2)} y_{i} y_{j}+C_{n i j k}^{(3)} y_{i} y_{j} y_{k}+\cdots
$$

where $y_{i}$ denotes the $i$ th component of $y$ and repeated index implies summation. The linear stability matrix $B$ and the nonlinear coupling coefficients $C$ 's are independent of $y$. If all nonlinear coupling coefficients identically vanish in equation (2), one has a purely linear dynamical system. Otherwise, it is a nonlinear dynamical system.

A linear coordinate transformation $x=T y\left(T \in \Re^{N \times N}\right)$ can always be performed and leads to a similarity transformation of the linear stability matrix $B$, i.e. $A=T B T^{-1}$ with $T^{-1}$ the inverse of $T$. In this paper we will insist on an orthogonal transformation $T$ to insure the orthogonality of the new basis $\hat{x}_{i}$ s. This is motivated by two considerations: 1] orthonormal basis are convenient for projecting higher order nonlinear terms; 2 ] the amplitude of the perturbation is expressed by norms, which is easy to relate in an orthonormal basis. The original dynamical system now takes the form 


$$
d x_{n} / d t=A_{n i} x_{i}+c_{n i j}^{(2)} x_{i} x_{j}+c_{n i j k}^{(3)} x_{i} x_{j} x_{k}+\cdots
$$

If $B$ commutes with its adjoint (transpose conjugate), i.e. $B$ is a normal matrix, then $A$ can be a diagonal matrix for a suitable orthogonal $T$ if the eigenvalues are distinct [1]. The diagonal elements of $A$ are the eigenvalues and determine both the eigenfrequency and the spectral stability of the system. If $B$ does not commute with its adjoint, the linear stability matrix is called non-normal [1] and $A$ generally can not be an exactly diagonal matrix with an orthogonal $T$. Instead $T$ is usually chosen so that $A$ is either in an up-triangle or a down-triangle form. A canonical example of such non-normal linear stability matrix $A$ is a Jordan block. For vanishing diagonal elements, the bifurcation scenario of the full nonlinear equation is studied in the literature under the name of Takens-Bogdanov bifurcation [2], following the seminal works of Takens [3] and Bogdanov [4]. Reference [2] also presents a number of physical applications that are characterized by Takens-Bogdanov bifurcation. We are particularly interested in a linear stability matrix of the Jordan-block form

$$
A \equiv\left(\begin{array}{cccc}
-a_{1} \epsilon & 1 & \ldots & 0 \\
0 & -a_{2} \epsilon & 1 & \vdots \\
\vdots & & \ddots & 1 \\
0 & \ldots & & -a_{N} \epsilon
\end{array}\right) .
$$

where all $a_{i}$ 's are positive and $0<\epsilon<1$ a small positive parameter that will be eventually set to approach zero in the asymptotic analysis.

Definition I.2 A nonlinear dynamical system with a linear stability matrix given in equation (4) is called a Takens-Bogdanov-type dynamical system. The generalized TakensBogdanov-type dynamical system can have non-vanishing entrees on all up-triangle elements of $A$.

In either case, the diagonal elements of $A$ are the eigenvalues and determine the spectral stability of the system. By definition, Takens-Bogdanov-type dynamical systems have the generic feature of monotonically approaching neutral stability as the control parameter $\epsilon$ goes to zero. 
It should be pointed out that a non-normal linear stability matrix does not imply nondiagonalisibility of the matrix. Non-diagonalisibility corresponds to an exact geometric degeneracy. In the case of equation (4), that requires an exact algebraic degeneracy, i.e. $a_{i}=a_{j}$ for $i \neq j$. Non-normality only implies a near or weak geometric degeneracy, i.e. the eigenvectors of the linear stability matrix are nearly parallel. We are mainly interested in those Takens-Bogdanov-type dynamical systems with diagonalizable linear stability matrices. The pathological case associated with a non-diagonalizable linear stability matrix makes a trivial example of the general results that we will show.

It is interesting to note that models of the Takens-Bogdanov-type have recieved particular attention recently in the study of transition to turbulence in parallel shear flows. Interested

readers are recommended to consult with a recent paper by Danchot and Manneville [5] for both an overview and a technical account of the competing schools of thoughts on that subject. Here we should concentrate on presenting the general theory of the finite amplitude instability in the class of mathematical models of the Takens-Bogdanov-type. Specific applications, such as that to the parallel shear flow transition problem, will be given elsewhere as it is more appropriate.

\section{Finite amplitude instability in Takens-Bogdanov-type dynamical systems}

Similar as the one dimensional example given earlier, a multi-dimensional normal system (i.e. an exact diagonal $A$ under an orthogonal $T$ ) typically has a normal scaling relationship $0<\alpha \leq 1$. This is more or less in line with the experimental observation that an equilibrium becomes more susceptible to noise as the system approaches neutral stability. Although an $\alpha>0$ is sufficient for the critical perturbation amplitude $\sigma_{c}$ to scale monotonically with linear stability parameter $\epsilon$ as it approaches neutral stability $\epsilon \rightarrow 0, \alpha=1$ actually separates two qualitative different regimes for the finite amplitude instability. For a normal $0<\alpha<1, \sigma_{c}$ decreases at a slower rate compared with that of $\epsilon$. If the scaling law is anomalous $\alpha>1, \sigma_{c}$ can shrink to zero at a rate much faster than that of $\epsilon$. Obviously, an anomalous scaling would 
imply that the finite amplitude instability might be playing a dominant role in determining the transitional properties in an experimental situation.

We will show that Takens-Bogdanov-type dynamical systems always have a positive scaling exponent, Corollary III.13. If the system is resonant, Definition III.7, the scaling exponent is strictly greater than one hence the finite amplitude instability is anomalous, Corollary III.10. A non-resonant Takens-Bogdanov-type dynamical system can also have a anomalous finite amplitude instability, provided that certain nonlinear terms exist in the expansion, Corollary III.14. The formulae to calculate the scaling exponent at the presence of arbitrary resonant and non-resonant nonlinear terms are given by Theorem III.9 and Theorem III.12.

As seen in the one dimensional example earlier, finite amplitude instability arrives due to a balance between the linear and leading nonlinear terms. This general principle equally applies to Takens-Bogdanov-type dynamical systems. The abnormality of the scaling law actually finds its root in the linearized dynamics rather than a pathology in the nonlinear terms. A prominent feature of the Takens-Bogdanov-type systems is the possibility of an exact or approximate geometrical degeneracy of two or more eigendirections of the linearized dynamics. The physical consequence of such degeneracy is the so-called transient amplification phenomena, sometimes also known as an algebraic instability. That is: an initial perturbation would grow (algebraically in time), rather than monotonically decay, in some linear subspace of the system despite its being spectrally stable in all directions. Of course this growth would eventually come to a halt and the perturbation starts to decay away, but only after it reaches to a sizable amplitude. This is why it is termed 'transient' amplifications.

Transient amplifications around the linear stable equilibrium force a separation of the asymptotic orders (in linear stability parameter $\epsilon$ ) between various participating components, which corresponds to an extremely elongated basin of attraction for the full nonlinear dynamics. Although nonlinearity is a necessity for the existence of a finite amplitude instability, it is the (approximate or exact) geometrical degeneracy of the linearized dynamics 
that changes the characteristics of the finite amplitude instability in Takens-Bogdanov-type dynamics. We will present an asymptotic method to treat the onset of the finite amplitude instability, which amounts to calculate the critical perturbation amplitude $\sigma_{c}$.

In addition to the possibility of an anomalous finite amplitude instability, the TakensBogdanov-type dynamical systems also respond differently to continous stochastic driving. Work in this area include that by Farrell and Ioannou [6], who studied the effect of stochastic forcing on a linearized Navier-Stokes equation around a shear flow, which is of Takens-Bogdanov-type but strictly linear. The effect of noise driving on a nonlinear TakensBogdanov-type system has been recently studied in [7] under the name of Takens-Bogdanov random walk.

The rest of the paper is organized as follows. Section II presents a two dimensional example to illustrate the geometrical view of the onset of a finite amplitude instability in a Takens-Bogdanov-type dynamical system, the so-called $O S A$ connection. The essence of the asymptotic analysis is also exposed in the same section. The $N$-dimensional problem is formulated and treated in section III. Specifically an alternative, more convenient, standard form of the Takens-Bogdanov-type dynamical system is given in section III A, along with the distinction between a resonant and a non-resonant nonlinear term. The resonant and non-resonant cases are then tackled separately in section III B and section III C.

\section{BASIC PICTURE: $O S A$ CONNECTION}

The abnormality of the scaling relationship characterizing the finite amplitude instability in Takens-Bogdanov-type systems comes from the interplay between nonlinearity and the geometrical degeneracy (or near-degeneracy) in linear order. The role of nonlinearity is to introduce a basin of attraction for the asymptotically stable solution. The geometrical degeneracy of the linearized dynamics, or in physics term, the transient amplification effect, forces the basin of attraction to be extremely elongated. It is the wide separation of scales of the basin of attraction in different directions that leads to the closeness of the basin boundary 

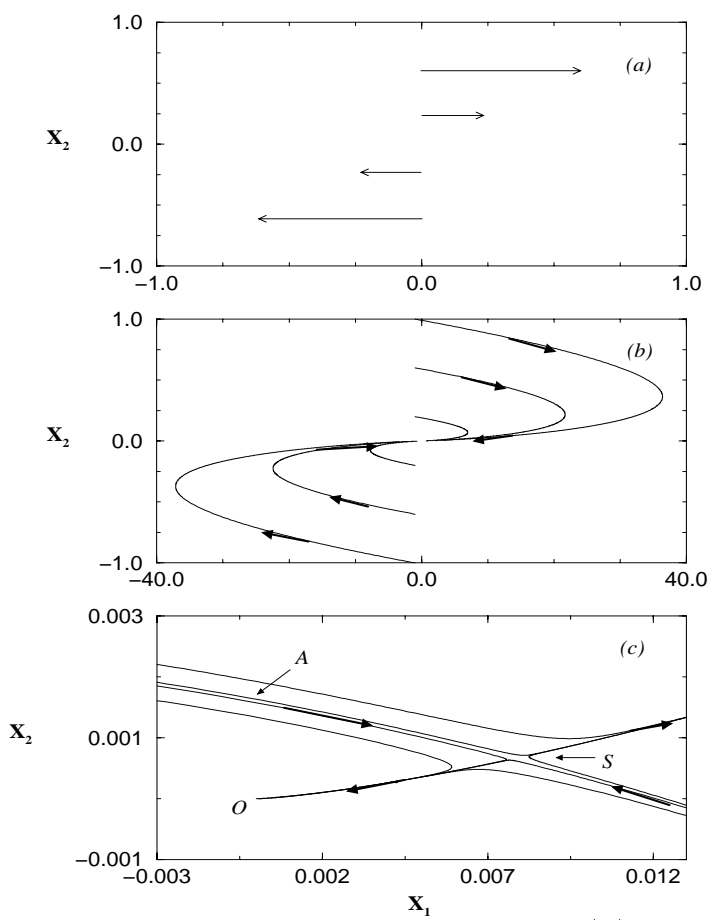

FIG. 2. Phase space plot of a $2 \mathrm{D}$ flow given by equations (1) and (2). (a) $\epsilon=a_{1}=a_{2}=0$, parallel shear flow; (b) $a_{1}=a_{2}=0, \epsilon=0.1$, degenerate node; (c). $a_{1}=a_{2}=1$ and $\epsilon=0.1$, basin of attraction and $O S A$ region ( $S$ is the saddle).

to the stable solution in a specific coordinate direction, and hence leads to the exponential scaling of the critical perturbation with the number of degrees of freedom participating in the transient amplification. This can be illustrated in the case of a two dimensional flow

$$
\begin{gathered}
\dot{x_{1}}=-\epsilon x_{1}+x_{2}+2 a_{1} x_{1}^{2} ; \\
\dot{x_{2}}=-\epsilon x_{2}+a_{1} x_{1} x_{2}+a_{2} x_{1}^{2} .
\end{gathered}
$$

At the absence of a nonlinear term $\left(a_{1}=a_{2}=0\right)$, the linear stable solution is a two dimensional degenerate node. The nonlinear terms as prescribed are the exact normal form up to quadratic order around a degenerate node in the limit of $\epsilon \rightarrow 0$. Bifurcation of nonlinear systems of this kind was first studied in [3,4], and bears the name of TakensBogdanov bifurcation. The underlying physics can be easily understood by examining the sequence of phase space changes as the parameters are varied.

If $\epsilon=a_{1}=a_{2}=0$, our example reduces to a divergence-free, parallel flow with constant shear, figure 2(a). A positive $\epsilon$ brings in dissipation and the origin becomes an asymptoti- 
cally stable solution, which is a degenerate node, figure 2(b). Still the system is stable for arbitrarily large perturbation. Typically, a trajectory starts on the $x_{2}$ axis at $x_{2}^{\prime}$, propagates almost parallel to the $x_{1}$ axis depending on how small $\epsilon$ is. It slows down as time $t \rightarrow 1 / \epsilon$ and makes a turn after reaching a distance $x_{2}^{\prime} / \epsilon$ along the $x_{1}$ axis. For $\epsilon \ll 1$ that corresponds to a large amplification of the initial perturbation of $1 / \epsilon \gg 1$ times. As the initial perturbation becomes greater, same behavior persists except the turning point being pushing further away from the origin. Now let the nonlinearity comes in with $a_{1}=a_{2}=1$. The most profound effect, as one would expect from a valid self-consistent model, is to impose an up-bound on the transient amplification factor of the linearized dynamics. It does so generally by introducing a basin of attraction. In the case of a Takens-Bogdanov-type system, a saddle point is brought in with its stable manifold forming part of the basin boundary and its unstable manifold connecting back to the origin on one side while escaping away on the other side, figure 2(c). What's also preserved from the linearized dynamics is the geometry in the so-called $O S A$ region where $O$ stands for the origin or the stable equilibrium, $S$ stands for the saddle or saddle-like point, and $A$ is the displacement of the basin boundary along the $x_{2}$ direction. Solving equations (5) and (6), one finds that the $x_{1 s} \approx \epsilon^{2}$ and $x_{2 A} \approx \epsilon^{3}$. Hence the critical perturbation $\sigma_{c} \propto \epsilon^{\alpha}$ with $\alpha=3$.

The distinction between the onset and the saturation of the finite amplitude instability can be clearly made based on the $O S A$ picture, figure 2(c). The critical perturbation amplitude is approximately given by where $A$ is. An initial perturbation lying between $O-A$ produces a trajectory approaching the saddle along its stable manifold and turns away from the saddle by following its unstable manifold to the stable equilibrium $O$. On the other hand, the system would diverge away by following the opposite branch of the unstable manifold of the saddle if the initial perturbation is beyond $A$. It is the $O S A$ connection that determines the onset of the finite amplitude instability. The saturation of the finite amplitude instability is on a different set of questions: what the new stable equilibrium is and what kind of route the system would follow to arrive at the new equilibrium. The $O S A$ picture provides the starting point for the saturation route but imposes no constraint on the characteristics of 
the new equilibrium. In other words, the onset and the saturation of the finite amplitude instability are separate issues and demands separate treatments.

One might feel that the $O S A$ connection is a peculiarity of the particular form of the example, equation (5). In actuality, the saddle-node connection is the topological signature of a Takens-Bogdanov bifurcation. In other words, the $O S A$ connection is a generic feature

for Takens-Bogdanov-type dynamical systems around the asymptotically stable solution.

\section{N-DIMENSIONAL PROBLEM: A MATHEMATICAL TREATMENT}

\section{A. Mathematical formulation}

Despite the similarity of the geometry of the trajectories, figure 2 (b) and (c), there is an important difference between the linear and nonlinear dynamics in the $O S A$ region, which is on the mechanism for the turning of a trajectory. In linear dynamics, the turn is made in $x_{1}$ direction which means $\dot{x_{1}}=0$ is the only constraint. The additional constraint for $\dot{x_{2}}\left(\dot{x_{2}}=0\right)$ was brought in by the nonlinear coupling in equation (6). That additional constraint transforms a moving turning point into a stagnation or fixed point (a saddle to be precise). Furthermore, transient amplification factor is determined by the turning condition, while equation (6) with nonlinear coupling determines the location of the saddle. This suggests that:

Proposition III.1 Once the location of the saddle is found from the last equation one can then combine it with the transient amplification factor from the turning condition to estimate the critical perturbation, i.e. the location of $A$.

The rest of the paper shows how to apply this idea to the general $N$-dimensional TakensBogdanov-type systems, equations (3,4). For the clarity of bookkeeping, the illustrative example will have $a_{1}=a_{2}=\cdots=a_{N}=1$ which corresponds to an $N$-dimensional 
degenerate node as the linear stable solution. ${ }^{1}$ It must be emphasized right away that neither the method of analysis nor the physical results depends on this exact degeneracy. An approximate degeneracy insures the validity of our asymptotics and leads to a rich set of behavior for the onset of the finite amplitude instability.

Another standard form of Takens-Bogdanov-type dynamical systems can make above statements mathematically rigorous. In appendix A, the following Lemma is shown.

Lemma III.2 Equations (3,4) can always be transformed via a nonlinear coordinate transformation, into the form

$$
\begin{gathered}
\dot{x_{1}}=-a_{1} \epsilon x_{1}+x_{2} ; \\
\dot{x_{2}}=-a_{2} \epsilon x_{2}+x_{3} ; \\
\dot{x_{3}}=-a_{3} \epsilon x_{3}+x_{4} ; \\
\vdots \\
\dot{x_{N}}=-a_{N} \epsilon x_{N}+f(x),
\end{gathered}
$$

where $f(x)$ is a polynomial of quadratic or higher order.

The new form has the triviality of limiting the nonlinear couplings to the $x_{N}$ component where the linear coupling with other degrees of freedom is absent. The simplicity of the linearized dynamics is maximumly preserved while the saddle condition associated with the full nonlinear dynamics concerns only one equation, equation (11). The nonlinear coordinate transformation generally destroy the orthonormality of the basis, but this is acceptable here since we will be mainly interested in scaling laws.

Imposing the turning condition $\dot{x}_{i}=0$ for $i=1, \cdots, N-1$, one finds an asymptotic ordering:

\footnotetext{
${ }^{1}$ An alternative notation is to have $a_{1} \sim a_{2} \sim \cdots \sim a_{N} \sim 1$. The aim is solely for the simplicity of bookkeeping, i.e. dropping all $a_{i}$ s in later discussions on scaling relationships.
} 
Lemma III.3 Around the turning point of the trajectory,

$$
x_{1} \gg x_{2} \approx \epsilon x_{1} \gg x_{3} \approx \epsilon^{2} x_{1} \gg \ldots \gg x_{N} \approx \epsilon^{N-1} x_{1}
$$

In other words, an initial perturbation of magnitude $\sigma$ in the $x_{N}$ component $x_{N}=\sigma$ would be amplified by $\epsilon^{i-N}$ times in the $x_{i}$ component and only one out of $N$ components is actually free. The asymptotic ordering of equation (12) transforms the original $N$-dimensional problem into an effectively one dimensional problem. Balancing the leading order terms in the remaining one dimensional system leads to an asymptotic estimates of the location of the saddle.

Proposition III.4 The nonlinear terms in equation (11) are ordered by two indexes. The first one is the order of the nonlinearity, $M$. The second index $L$ is the asymptotic order in $\epsilon$.

Definition III.5 By an Mth order nonlinear term of asymptotic order $\epsilon^{L}$, or $(M, L)$-order term, we refer to a monomial of the form $\prod_{i=1}^{N} x_{i}^{a_{i}}$ that satisfies

$$
\sum_{i=1}^{N}(i-1) a_{i}=L \text { and } \sum_{i=1}^{N} a_{i}=M .
$$

with $a_{i}$ s non-negative integers.

In calculating the asymptotic order of an arbitrary monomial we have substituted the ordering relations of the linear terms, equation (12), and converted a multivariate monomial into a single variable monomial. At the presence of only an $(M, L)$-order nonlinear term, equation (11) takes the form

$$
\dot{x_{N}}=-\epsilon^{N} x_{1}+\epsilon^{L} x_{1}^{M} .
$$

The saddle condition $\dot{x_{N}}=0$ gives an estimate of the location of the saddle point.

Lemma III.6 The $x_{1}$ component of the saddle point associated with an $(M, L)$-order nonlinear term obeys

$$
x_{1}^{s} \approx \epsilon^{\frac{N-L}{M-1}}
$$


Two entirely different cases have to be separated for further discussion depending on whether $L$ is smaller than $N$.

Definition III.7 The nonlinear terms with $L<N$ are resonant terms while those with $L \geq N$ are non-resonant terms.

The terminology is borrowed from normal form theory and it can be easily seen why. The emergence of a new equilibrium, the saddle point, represents a topological change in the vicinity of the original equilibrium from a perturbative point of view. A nonlinear term with $L \geq N$ gives rise to a new equilibrium at $x_{1} \geq 1$. This is not consistent with the spirit of perturbation since the expansion series is divergent at $x_{1} \geq 1$. As far as a perturbative analysis is concerned, these nonlinear terms are not the important ones in the expansion series. In the normal form theory they are the non-resonant terms which can be removed by a coordinate transformation in the limit of neutral stability $\epsilon=0$. In contrary, a nonlinear term with $L<N$ insures that the largest component of the system $x_{1}^{s}$ is less than one and approaches zero as $\epsilon \rightarrow 0$. These are the resonant nonlinear terms responsible for the topological changes in the close proximity of the original equilibrium.

Proposition III.8 For the purpose of estimating the onset of the finite amplitude instability in Takens-Bogdanov-type dynamical systems, all non-resonant terms can be ignored as long as there is at least one resonant term permitted by $f(x)$ in equation (11), independent of how high the nonlinear order $M$ might be.

The exceptional case that enough symmetry in the physical systems excludes resonant terms from $f(x)$ to arbitrarily high order requires an explicit consideration of the nonresonant terms. Even in that case, the relative importance of each non-resonant in determining the onset of the finite amplitude instability is straightforwardly determined by the asymptotic analysis. 


\section{B. Case I: the nonlinear term is resonant}

Let's first consider the resonant case, i.e. there is at least one term with $L<N$. In the general resonant case, $L$ can take any integer value from 0 to $N-1$. One can introduce a new index $L^{*}=N-L$. With the new index, the $x_{1}^{s}$ is at

$$
x_{1}^{s} \approx \epsilon^{\frac{L^{*}}{M-1}} \text { with } L^{*}=1,2, \cdots, N
$$

The shortest distance between the original asymptotically stable equilibrium (the origin) to

its basin boundary is approximately along the $x_{N}$ direction and $\epsilon^{N-1}$ times of $x_{1}^{s}$ (the inverse process of transient amplification),

$$
\sigma_{c} \approx \epsilon^{N-1+L^{*} /(M-1)}
$$

Theorem III.9 In a resonant $N$-dimensional Takens-Bogdanov-type dynamical system, a resonant nonlinear term of order $(M, L)$ gives a finite amplitude instability of scaling exponent

$$
\alpha=N-1+\frac{L^{*}}{M-1}
$$

with $L^{*} \equiv N-L=1,2, \cdots, N$.

The scaling relation presented earlier by Tracy and Tang [8] is the limiting case that $L^{*}=N$,

$$
\sigma_{c}(L=N) \approx \epsilon^{N-1+N /(M-1)} .
$$

This result demonstrates that the scaling laws $\sigma_{c} \approx \epsilon^{\alpha}$ is always anomalous in that $\alpha>1$ for a Takens-Bogdanov-type dynamical system as long as there is at least one resonant nonlinear term. It is obvious that $\alpha=N-1+\frac{L^{*}}{M-1}$ reaches its minima for the smallest $N$ and $L^{*}$, but largest $M$. The smallest $N$ is 2 . The smallest $L$ is 1 . For arbitrarily high but finite nonlinearity, $1 /(M-1)$ is positive. Hence $\alpha_{\min }>1$.

Corollary III.10 The finite amplitude instability of a resonant nonlinear TakensBogdanov-type dynamical system is always anomalous. 


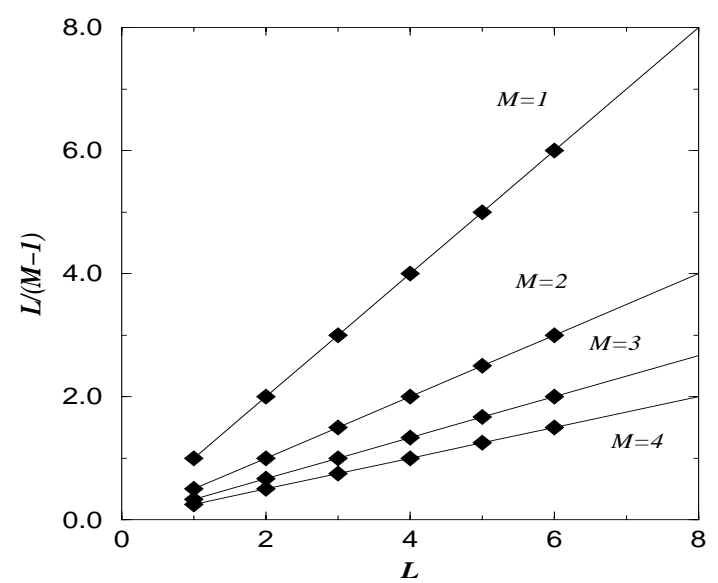

FIG. 3. Dominance crossing: the importance of a nonlinear term is determined by both its order $M$ and the mode number $L$ where it feeds back.

Our result for the scaling relations of the finite amplitude instability also suggests the possibility for dominant resonant terms to cross over different orders of nonlinearity $(M)$. To see that, one can plot $y=x /(M-1)$ for integer $M>1$ and study the value for $y$ at $x=1, \cdots, N$. The relative importance of $(M, L)$-order nonlinear term is obvious from this plot, figure 2. It also provides the guideline in the analysis of finite amplitude instabilities in resonant Takens-Bogdanov-type dynamical systems.

\section{Case II: the nonlinear terms are non-resonant}

Let's first emphasize again that it is an exceptional case for all resonant nonlinear terms to vanish. This is especially difficult if a Taylor expansion of $f(x)$ gives an infinite series. Stringent symmetry constraints must present to exclude all resonant nonlinear terms. Nevertheless, if it does occur, the onset of the finite amplitude instability can have markably different behavior.

For a non-resonant Takens-Bogdanov-type dynamical system, the asymptotic order of the nonlinear term $L$ is always no less than the degrees of freedom $N$ that participate in transient amplification. We can introduce a new index $L^{\prime}=L-N$ which is a non-negative integer in the non-resonant case. As usual we assume the coupling coefficients are independent of $\epsilon$. 
(An exotic $\epsilon$ dependence of some coefficients is possible in applications but does not make an intelligent example problem).

Proposition III.11 The highest asymptotic order is archived by a nonlinear term of the form $x_{N}^{M} \approx \epsilon^{M N-M}$. That gives the up-bound for $L^{\prime}, L_{\max }^{\prime} \equiv M N-M-N$. The lowest bound for $L^{\prime}$ is, of course, $L_{\min }^{\prime}=0$.

Following the analysis in last section, the critical perturbation amplitude corresponding to an $\left(M, L^{\prime}\right)$ non-resonant nonlinear term is given by

$$
\sigma_{c} \approx \epsilon^{N-1-L^{\prime} /(M-1)} \text { with } L^{\prime}=0,1, \cdots, M N-M-N
$$

Theorem III.12 The finite amplitude instability in a non-resonant Takens-Bogdanov-type dynamical system has a scaling exponent

$$
\alpha=N-1-\frac{L^{\prime}}{M-1}
$$

corresponding to a non-resonant nonlinear term of order $(M, L)$ with $L^{\prime} \equiv L-N$ taking the value $0,1, \cdots, M N-M-N$.

The fundamental difference between equation (14) and equation (15) is the negative sign in front of the nonlinear correction term $L^{\prime} /(M-1)$. That is precisely why all non-resonant terms are negligible in determining the onset of the finite amplitude instability if there is one single resonant term in the full dynamics, Proposition III.8.

A few more comments can be made based on equation (15). First the scaling exponent is always positive since the scaling exponent $\alpha$ has its minimum when $L^{\prime}=L_{\max }^{\prime} \equiv M N-$ $M-N$,

$$
\alpha_{\min }=\frac{1}{M-1}>0, \text { with } M=2,3,4, \cdots
$$

Corollary III.13 The finite amplitude instability in a non-resonant Takens-Bogdanov-type dynamical system has a positive scaling exponent. 
Secondly, both normal and anomalous scaling relations are possible for a non-resonant Takens-Bogdanov-type dynamical system. The critical exponent $\alpha_{c}=1$ is achieved if there is a non-resonant nonlinear term $\left(L_{c}^{\prime}, M\right)$ satisfying

$$
L_{c}^{\prime}=L_{\max }^{\prime}-M+2
$$

Here $L_{\max }^{\prime} \equiv M N-M-N$ is a function of the number of degenerate or near-degenerate modes and the order of the nonlinearity.

Corollary III.14 The finite amplitude instability in a non-resonant Takens-Bogdanov-type dynamical system is anomalous if there is a non-resonant $\left(L^{\prime}, M\right)$-order nonlinear term with $L^{\prime}<L_{c}^{\prime} \equiv L_{\max }^{\prime}-M+2$

If the highest nonlinear term is quadratic, the critical exponent $\alpha_{c}=1$ is obtained at the presence of a non-resonant term with the highest possible asymptotic order.

The difference between a resonant Takens-Bogdanov-type dynamical system and a nonresonant one can also be thought of as a geometrical one. For a resonant Takens-Bogdanovtype dynamical system, the basin of attraction enclosed by the $O S A$ region shrinks from all directions at the system approaches neutral stability $(\epsilon \rightarrow 0)$. In contrary, if a nonresonant system is approaching neutral stability, the $O S A$ region shrinks in some direction but expands in other coordinates. A resonant Takens-Bogdanov-type dynamical system always has an anomalous scaling relations for the onset of the finite amplitude instability. A non-resonant Takens-Bogdanov-type dynamical system has an anomalous scaling relation if the inequality

$$
L^{\prime}<L_{\max }^{\prime}-M+2
$$

is satisfied by at least one non-resonant term in the dynamics. Otherwise it has a normal scaling relationship for the onset of the finite amplitude instability. 


\section{CONCLUSIONS}

The onset of a finite amplitude instability is studied in nonlinear Takens-Bogdanovtype dynamical systems. which are divided into two groups: non-resonant or resonant. A resonant Takens-Bogdanov-type dynamical system always has an anomalous finite amplitude instability. Resonant Takens-Bogdanov-type dynamical system is the generic one since the system is resonant if there exists one single resonant term. A non-resonant Takens-

Bogdanov-type dynamical system can have both normal and anomalous finite amplitude instabilities, depending on whether at least one non-resonant term satisfying inequality (16) exists.

\section{ACKNOWLEDGEMENTS}

We would like to thank Gene Tracy for useful discussions.

\section{APPENDIX A: PROOF OF LEMMA III.2}

The peculiar structure of a Jordan block allows another general form of equation (3). The new form has only linear coupling in the first $N-1$ modes that participate in transient amplification. All nonlinear couplings are retained in the last equation where no linear coupling with other degrees of freedom exists. To see that, let's write out equation (3) explicitly with $A$ a Jordan block as in equation (4),

$$
\begin{array}{r}
\dot{x_{1}}=-a_{1} \epsilon x_{1}+x_{2}+f_{1}(x) ; \\
\dot{x_{2}}=-a_{2} \epsilon x_{2}+x_{3}+f_{2}(x) ; \\
\dot{x_{3}}=-a_{3} \epsilon x_{3}+x_{4}+f_{3}(x) ; \\
\vdots \\
\dot{x_{N}}=-a_{N} \epsilon x_{N}+f_{N}(x),
\end{array}
$$

where $f_{s}$ are polynomials of quadratic or higher. 
A nonlinear coordinate transformation $x_{2}^{\prime}=x_{2}+f_{1}(x)$ removes the nonlinear coupling in the first component, equation (A1),

$$
\begin{array}{r}
\dot{x_{1}}=-a_{1} \epsilon x_{1}+x_{2}+f_{1}(x) ; \\
\dot{x_{2}^{\prime}}=-a_{2} \epsilon x_{2}^{\prime}+x_{3}+f_{2}^{\prime}(x) ; \\
\dot{x_{3}}=-a_{3} \epsilon x_{3}+x_{4}+f_{3}(x) ; \\
\vdots \\
\dot{x_{N}}=-a_{N} \epsilon x_{N}+f_{N}(x),
\end{array}
$$

Similarly another nonlinear coordinate transformation $x_{3}^{\prime}=x_{3}+f_{2}^{\prime}(x)$ removes the nonlinear coupling in the second component, equation (A7). A total $N-1$ operations remove all nonlinear couplings from the first $N-1$ equations. Dropping all the primes, equation (3) now takes the form

$$
\begin{array}{r}
\dot{x_{1}}=-a_{1} \epsilon x_{1}+x_{2} ; \\
\dot{x_{2}}=-a_{2} \epsilon x_{2}+x_{3} ; \\
\dot{x_{3}}=-a_{3} \epsilon x_{3}+x_{4} ; \\
\vdots \\
\dot{x_{N}}=-a_{N} \epsilon x_{N}+f(x)
\end{array}
$$

with $f(x)$ consisting of polynomials of quadratic order or higher. This is the desired alternative standard form. 


\section{REFERENCES}

[1] F. W. Bryon, Jr. and R. W. Fuller, Mathematics of classical and quantum physics (Dover, New York, 1992).

[2] J. Guckenheimer and P. Holmes, Nonlinear oscillations, dynamical systems and bifurcations of vector fields (Springer-Verlag, New York, 1983).

[3] F. Takens, Publ. Math. I. H. E. S. 43, 47 (1974).

[4] R. I. Bogdanov, Func. Anal. Appl. 9, 144 (1975).

[5] D. Danchot and P. Manneville, J. Phys. II France 7, 371-389 (1997), and references therein.

[6] B.F. Farrell and P.J. Ioannou, Phys. Rev. Lett. 721188 (1994).

[7] E.R. Tracy, X.Z. Tang, and C. Kulf, Phys. Rev. E 573749 (1998).

[8] E. R. Tracy and X. Z. Tang, Physics Letters A 242 239-244 (1998). 